\title{
MPC 2.0๑, software para la aplicación del método AHP de toma de decisiones multicriterio
}

Recibido: 3 marzo 2011 / Aceptado: 27 xullo 2011

(c) IBADER- Universidade de Santiago de Compostela 2011

Resumen En este trabajo se presenta el software MPC $2.0^{\odot}$, diseñado para facilitar la aplicación de la metodología de toma de decisiones AHP (Analytic Hierarchy Process), basada en la comparación por pares. El programa resulta especialmente útil en las decisiones en las que sea necesario considerar numerosos y diferentes tipos de criterios (cuantificables o no) y/o muchas posibles alternativas, así como también cuando sea preciso tener en cuenta diferentes repeticiones de la decisión de uno o diferentes usuarios. MPC 2.0॰ facilita las comparaciones por pares de los criterios (primera fase de la metodología) y de las alternativas bajo cada criterio (segunda fase), minimizando los sesgos implícitos a las mismas. Permite establecer un esquema de criterios en dos niveles (criterios y subcriterios, con la posibilidad de 40 elementos en cada uno) y la realización independiente de la comparación de los criterios y de las alternativas. MPC $2.0^{\circ}$ automatiza todos los cálculos matriciales posteriores para asignar el peso de cada alternativa y calcula el grado de inconsistencia de la decisión (indica si la calificación establecida es lógica o no). MPC 2.0 $0^{\circ}$ integra un interface gráfico para facilitar la exposición de los resultados, así como un análisis de sensibilidad, que ofrece al usuario información de la variación de los resultados al modificar los pesos de los criterios.

Palabras clave Comparación por pares, Criterios, Alternativas

Fernando Pérez Rodríguez · Alberto Rojo Alboreca

Unidade de Xestión Forestal Sostible (UXFS). Departamento de

Enxeñaría Agroforestal. Escola Politécnica Superior de Lugo.

Universidade de Santiago de Compostela. Campus Universitario

s/n 27002 Lugo (Spain).

Tel: $982823222 / 1$

e-mail: fernando.perez.rodriguez@usc.es
Abstract This paper presents the MPC $\odot 2.0$ software designed to facilitate the implementation of the methodology known AHP (Analytic Hierarchy Process), based in multicriteria decision making under pairwise comparison. The software is especially useful in decisions where it is necessary to consider many different types of criteria (both readily quantifiable or not) and / or many possible alternatives, as well as when it is necessary to consider different instances of the decision by the same or different users. MPC $\odot 2.0$ provides pairwise comparisons of criteria (first phase of the methodology) and the alternatives under each criterion (second phase), minimizing the implicit bias to them. It also allows the scheme of a two-levels criteria (criteria and subcriteria, with the possibility of 40 items each) and conducting independent pairwise comparison of criteria and alternatives under each criterion. From these comparisons MPC $\odot 2.0$ automates all matrix calculations to assign the weight (or priority) of each alternative, and calculates the degree of inconsistency of the decision (which indicates whether the set rating is logic). MPC ${ }^{\odot} 2.0$ integrates a graphical interface to facilitate the presentation of the results, as well as a sensitivity analysis, which provides the user with the variation of the results by modifying the weights of the criteria.

Key words Pairs comparion, Criteria, Alternatives

\section{Introducción}

Existen muchas metodologías de análisis multicriterio que sirven como ayuda o apoyo en la toma de decisiones complejas o de cierta dificultad, algunas de ellas estudiadas por Buongiorno \& Keith (2003), Kangas \& Kangas (2005) o Pena de Ladaga y Berger (2006), pero es sin duda la metodología AHP o Analytic Hierarchy Process (Saaty, 1980) la más utilizada a la hora de establecer una prioridad o jerarquía de alternativas (o conjunto de soluciones factibles) para la consecución de la meta marcada. En la metodología AHP las alternativas son evaluadas bajo unos criterios que se tienen en cuenta en la decisión (como por ejemplo: calidad, cualidad, tamaño, durabilidad, etc), 
pudiendo ser éstos cualitativos o cuantitativos, lo que constituye una de las grandes virtudes de este método.

Han sido muchos los autores que han estudiado el AHP, como Toskano y Bruno (2000), Zanazzi (2003) o Schoner \& Wedley (2007), entre otros muchos, que se ha aplicado para la toma de decisiones complejas en todo el mundo y en distintos campos de actividad empresarial, gubernamental, educativa, etc.

El método AHP consiste, a grandes rasgos, en los cuatro siguientes pasos: i) Definir una jerarquía de decisión con niveles, incluyendo el objetivo en el nivel 1 , los criterios implicados en la toma de decisiones en el nivel 2 y las alternativas posibles en el nivel 3. ii) Estimar pesos o preferencias de los criterios (nivel 2) comparándolos por pares. iii) Comparar las alternativas (nivel 3) por parejas utilizando cada criterio (nivel 2) por separado. iv) Seleccionar la mejor alternativa.

La emisión de juicios para el establecimiento de los pesos se realiza por comparaciones pareadas, esto es, se determina primero el peso o importancia de cada uno de los criterios mediante su enfrentamiento por pares en todas las posibles combinaciones, estableciéndose la preferencia en cada par mediante una escala discreta, como por ejemplo la propuesta por Saaty (1980) con valores entre 1 (misma importancia) y 9 (absoluta importancia).

Una vez establecido el peso de los criterios dentro de una decisión, se estima el peso de cada alternativa realizando el mismo proceso y utilizando la misma escala anteriormente mencionada, pero esta vez se enfrentan las alternativas por separado bajo cada criterio. A partir de todas esas comparaciones pareadas, una serie de cálculos matriciales permiten obtener el peso o prioridad final de las alternativas consideradas, además de un grado de inconsistencia, esto es, un valor que indica si la calificación establecida es lógica, o si por el contrario tiene cierta inconcordancia. Esta inconsistencia se calcula en base a la valoración de la homogeneidad de las matrices normalizadas con las matrices de comparación, y según Saaty (1990), una matriz de comparaciones se muestra consistente, y con ello válida, si este grado de inconsistencia es menor de 0,1.

Para la aplicación de esta metodología existen varios programas informáticos, siendo Expert Choice (www.expertchoice.com) el más conocido a nivel mundial. En este trabajo se presenta el software MPC ${ }^{\odot}$ en su versión 2.0, que ha sido desarrollado por los autores para el estudio y mejora de esta metodología, buscando minimizar diversos sesgos que suelen ocurrir cuando un decisor emite un juicio u opinión (en este caso, cuando realiza una comparación por pares de criterios o alternativas).

Por otra parte, existen numerosos ejemplos de aplicación del AHP en muchos campos, pero específicamente se pueden encontrar aplicaciones en el contexto forestal y del desarrollo rural en Leskinen \& Kangas (1998), Gadow \& Bredenkamp (1992), Kangas et al. (2001), Schmoldt et al. (2001), etc. En este trabajo se presenta un ejemplo de aplicación del AHP adaptado a las condiciones y necesidades forestales del medio rural gallego, cuyo objetivo es introducir una batería de criterios de tipo ambiental, social, económico y técnico en una decisión real de gestión bajo un marco de limitaciones económicas, y obtener un acercamiento a una solución óptima minimizando los costes de inventario, análisis y proceso de datos.

\section{Material y métodos}

\section{El software MPC॰}

Para la aplicación de esta metodología se ha desarrollado el software MPC ${ }^{\odot}$, que automatiza todo el proceso analítico jerárquico. Partiendo de la primera versión del programa (1.0) y de diversos estudios y ensayos, se ha desarrollado la versión 2.0, utilizando en ambos casos el lenguaje VB.Net bajo Microsoft .NET Framework versión 4.0 (x86). La utilización y desarrollo de esta herramienta tiene el objetivo de ser la base para el estudio de la metodología AHP, quedando esta última versión como Beta (o en desarrollo) para uso exclusivo de investigación, aunque posteriormente se publicará, al igual que la versión 1.0, bajo una licencia de uso a determinar.

\section{Características genéricas de la serie MPC $\odot$}

En MPC $\odot$ se ha implementado un proceso de automatización de exposición de pares para su comparación o calificación. El proceso de automatización varía si se están calificando criterios u alternativas. En el primer caso, se comparan primero los criterios del primer nivel, y posteriormente y terminada esta fase, los criterios de los distintos subniveles de manera independiente. Con ello se obtiene una matriz de comparaciones para el primer nivel, y tantas matrices de comparaciones como subniveles haya. La aplicación del cálculo matricial dará como resultado el peso medio obtenido para cada criterio, estando este definido dentro del intervalo $[0,1]$.

En el caso de la valoración de las alternativas, el proceso de automatización difiere del de la calificación de criterios, puesto que solo se realiza en los extremos del esquema; esto es, si un criterio se divide en subcriterios, se efectúa la comparación de pares de cada alternativa bajo cada uno de los subcriterios. En caso de que los criterios no se dividan en subcriterios, esa comparación se efectúa bajo el criterio raíz.

El módulo de aleatoriedad que incorpora MPC ${ }^{\odot}$ permite exponer los pares de manera que la aparición de los criterios o las alternativas a izquierda o derecha sea aleatoria en la ventana donde se realiza la comparación. Se pretende con ello evitar que en diversas repeticiones pueda intervenir la memoria visual, lo cual causaría sesgos en la emisión del juicio, desembocando en un resultado poco útil. Para ello se genera un número aleatorio y se resta con otro del mismo rango de amplitud; en el caso de resultar un número positivo, se asocia al valor de 0 , y si resulta uno negativo al valor de 1 , variando en un caso $u$ otro la disposición del criterio a izquierda o derecha de manera 
aleatoria, por lo que en cada repetición será diferente la localización de los criterios en la pantalla de comparación. Además, el proceso de automatización permite focalizar la atención en el par que se está calificando, puesto que permite visualizar un par de manera independiente en pantalla, sin necesidad de elegir previamente en la matriz qué par va a ser calificado.

Por otra parte, la división en subniveles permite que un nivel principal de criterios pueda ser dividido en subcriterios, con el objetivo de facilitar al usuario la fragmentación de un criterio, evitando así su generalización.

\section{Desarrollo de mejoras especificas entre las versiones} 1.0 y 2.0 de MPC $\odot$

Con respecto a la anterior versión (1.0), se han ampliado las siguientes capacidades:

- Esquema de criterios: Posibilidad de introducir esquemas de criterios en dos niveles (criterios y subcriterios), estableciéndose una limitación inicial de 40 criterios raíz que a su vez se pueden dividir en 40 subcriterios.

- Exposición de resultados mediante gráficos: Se incorpora una ventana para visualizar los resultados en gráficas de líneas o barras (según elección del usuario), lo que facilita su interpretación.

- Bases de datos: Se ha incorporado una base de datos local desarrollada en SQLite (para Microsoft .NET Framework versión 4.0) donde se registran los resultados que podrán ser cargados posteriormente. Además se pueden crear nuevas bases para independizarlas de la base local y poder ser enviadas a otros usuarios.

- Diferentes usuarios: La versión 2.0 está capacitada para poder registrar varios usuarios para una misma decisión.

- Análisis de sensibilidad: Se ha incorporado la capacidad de realizar un análisis de sensibilidad, que consiste en determinar los cambios que se producen en los resultados al variar la magnitud del peso de un determinado criterio. Para ello es necesario conocer las relaciones entre criterios, para establecer como varían al cambiar alguno de sus pesos. Sin embargo, existe gran dificultad para conocer la existencia de tales relaciones y para cuantificarlas, por lo que es necesario aplicar otros métodos como los expuestos por Triantaphyllou \& Sanchez (1997) o Saletlli et al. (2006):

a) Variar los pesos proporcionalmente: consiste en definir una relación entre criterios o alternativas de manera proporcional a su peso, pero el problema que subyace de estas relaciones proporcionales es que no está clara la justificación de por qué un criterio que tiene más peso que otro tenga que variar en mayor proporción que otros que tengan menos peso, y viceversa.

b) Variar los pesos aritméticamente: este método se basa en disminuir o aumentar los pesos de los criterios o alternativas una magnitud constante y dependiente de la diferencia del peso que se pretende variar. Por ejemplo, si se aumenta el peso de un criterio un $30 \%$, los demás criterios reducen su peso equitativamente en una magnitud constante, en este caso $30 /(n-1)$, siendo $n$ el número de criterios. La problemática de este método surge cuando los pesos se hacen negativos, puesto que no es lógico asumir que un criterio tenga un peso menor que cero (aunque matemáticamente tenga su justificación, puesto que el sumatorio de los pesos de los criterios ha de ser 1), además de que se ha de cumplir el principio de reversibilidad, que no se cumpliría si llegado a 0 el criterio no se contempla en la variación.

El método de variación finalmente adoptado ha sido el de variación proporcional de los pesos. Sin embargo, se hace patente la dificultad de aplicar un análisis de sensibilidad bajo esta metodología en una repetición, aunque sí es posible aplicar un análisis de variaciones entre varias repeticiones, pudiendo observarse cual es el rango de variación que un decisor tiene sobre un criterio o sobre una alternativa bajo el factor tiempo, o comparar diversas repeticiones entre usuarios.

\section{Aplicación práctica}

En este trabajo se ha realizado una aplicación práctica que corresponde al asesoramiento a un propietario forestal que posee 6 parcelas y quiere desbrozarlas teniendo en cuenta que, debido a limitaciones económicas, solo puede desbrozar dos al año. Se busca, por tanto, establecer la prioridad de las parcelas para ser desbrozadas, minimizando además el riesgo de una posible sanción administrativa por abandono de las fincas en un posible incendio forestal. Para ello, el primer paso consiste en establecer los criterios que serán evaluados, que se han organizado en dos niveles, agrupándolos en criterios económicos, ambientales, sociales y técnicos:

\section{Criterios económicos:}

a) Posibilidad de sanción administrativa: según la normativa actual aplicable, la Ley $3 / 2007$, de 9 de abril, de prevención y defensa contra los incendios forestales de Galicia, es obligatorio desbrozar los terrenos de monte próximos a viviendas, estableciéndose un régimen sancionador.

b) Rendimiento: Depende de la dificultad que existe en la parcela para efectuar el desbroce, como puede ser el ancho de calle, densidad de la masa, altura de poda, especies con régimen de protección que requieren un desbroce selectivo, etc.

c) Mecanizable: Se entiende por mecanizable aquella parcela que reúne las condiciones para poder realizar la tarea de desbroce mediante tractor con desbrozadora de cadenas o martillos.

\section{Criterios ambientales:}

a) Erosión: Riesgo de que se pueda producir algún tipo de erosión y su magnitud, en el caso de llevar a cabo el desbroce de la parcela, debido a la pendiente.

b) Biodiversidad: Riesgo de que se pierda o modifique la biodiversidad del sistema silvícola por efectuar el desbroce en la parcela evaluada. 


\section{Criterios sociales}

a) Recreo: Existencia de zonas de paseo o tránsito de excursionistas cercanos o en la propia parcela a desbrozar.

b) Otros usos: Situación en una zona de pastoreo, de caza u otros usos, los cuales se pueden ver afectados por un desbroce en la zona.

c) Cercanía a viviendas: Riesgo de una posible continuidad de un incendio desde la parcela hacia viviendas cercanas.

\section{Criterios técnicos}

a) Altura del matorral: Criterio muy importante por la necesidad de desbroce para minimizar el riesgo de incendios en la parcela disminuyendo la continuidad vertical o la competencia entre especies.

b) Carga del matorral: Relacionado con la posible densidad de matorral o monte bajo, y la necesidad de desbroce para minimizar la posible intensidad de un incendio forestal o la competencia entre especies.

c) Necesidad de poda: Relacionado con la necesidad de desbroce para facilitar la accesibilidad a los pies para proceder a su poda.

d) Cercanía al turno: Disminuir la carga de matorral para aumentar el rendimiento del aprovechamiento maderero, incluyendo corta y saca.

Las alternativas en este ejemplo son las seis parcelas, en las que se ha realizado un inventario visual, recogiendo información que permita el establecimiento de los pesos comparativos entre criterios y alternativas al utilizar el programa. Un resumen del inventario de campo realizado en las parcelas se puede ver en la Tabla 1.

\begin{tabular}{|c|c|c|c|c|c|c|}
\hline & Parcela 1 & Parcela 2 & Parcela 3 & Parcela 4 & Parcela 5 & Parcela 6 \\
\hline Superficie & 0,517 ha & 1,034 ha & 0,698 ha & 1,103 ha & 0,711 ha & 0,576 ha \\
\hline Pendiente media & $20,2 \%$ & $6,4 \%$ & $10,8 \%$ & $13,9 \%$ & $11,5 \%$ & $9,4 \%$ \\
\hline Pedregosidad & $30 \%$ & $15 \%$ & $10 \%$ & $25 \%$ & $10 \%$ & $15 \%$ \\
\hline Especie principal & Quercus robur & $\begin{array}{l}\text { Pinus } \\
\text { pinaster }\end{array}$ & $\begin{array}{l}\text { Pinus pinaster y } \\
\text { Quercus robur }\end{array}$ & $\begin{array}{l}\text { Pinus } \\
\text { pinaster }\end{array}$ & $\begin{array}{c}\text { Pinus pinaster } \\
\text { y Quercus } \\
\text { robur }\end{array}$ & $\begin{array}{l}\text { Pinus pinaster y } \\
\text { Quercus robur }\end{array}$ \\
\hline $\begin{array}{l}\text { Edad aprox. (años) } \\
\text { de la esp. principal }\end{array}$ & - & $9-10$ & $6-7$ & $17-20$ & Irregular & Irregular \\
\hline Carga de matorral & Alta & Media & Media & Alta & Alta & Alta \\
\hline $\begin{array}{l}\text { Grado de } \\
\text { mecanización } \\
\text { posible }\end{array}$ & Bajo & Alto & Bajo & Medio & Medio & Medio \\
\hline $\begin{array}{c}\text { Proximidad a } \\
\text { viviendas }\end{array}$ & Si $(200$ y $250 \mathrm{~m})$ & $\mathrm{Si}$ & No & No & No & Si $(150$ y $200 \mathrm{~m})$ \\
\hline Acceso por pista & $\mathrm{Si}$ & $\mathrm{Si}$ & $\mathrm{Si}$ & $\mathrm{Si}$ & $\mathrm{Si}$ & $\mathrm{Si}$ \\
\hline
\end{tabular}

Tabla 1.- Descripción de las parcelas

Una vez conocidos los criterios y alternativas, se ha procedido a introducir el esquema de la decisión en el programa MPC $\odot 2.0$ para proceder a su evaluación, primero comparando por pares los criterios y posteriormente comparando, también por pares, las alternativas bajo cada criterio, aunque en el software se puedan efectuar de manera independiente cada una de estas etapas. Para aumentar la precisión de los resultados, se han repetido cinco veces las calificaciones de criterios y una la calificación de alternativas. Estas repeticiones se han efectuado de manera independiente y separadas en intervalos de tiempo, con el fin de minimizar los efectos de memoria visual, que podrían crear prejuicios o emisión de juicios sesgados. En este caso solo ha intervenido un decisor, pero se podría hacer intervenir a otros decisores, lo que aportaría al proceso mayor cantidad de resultados a evaluar, pudiéndose cuantificar y comparar calificaciones de las distintas personas.

\section{Resultados}

Los pesos obtenidos por cada criterio en la comparación por pares realizada mediante el programa MPC $\odot$ en la aplicación práctica, así como su coeficiente de variación, se muestran en la Tabla 2, mientras que los de la posterior comparación por pares de las alternativas bajo cada criterio se muestran en la Tabla 3 . Se puede observar que el coeficiente de variación de los pesos obtenidos es bajo, lo que significa muy poca dispersión de los resultados y, por lo tanto, una opinión homogénea. Además, en dichas Tablas 2 y 3 se muestran, entre paréntesis, las inconsistencias encontradas. Como ya se ha indicado, se puede asumir que un valor menor de 0,1 significa que se está tomando una decisión consistente.

MPC॰ 2.0 incluye múltiples posibilidades de cálculo matricial para obtener los resultados generales de todo el proceso. En este caso se ha decidido interaccionar todas las repeticiones de los pesos de evaluación de criterios con la repetición de evaluación de alternativas bajo cada criterio, con el objetivo de poder apreciar las diferencias entre ellas como se puede observar en la Figura 1. A la hora de establecer una inconsistencia, el software utilizado se basa en el análisis de las repeticiones realizadas, pues aunque teóricamente se puede obtener una inconsistencia de las matrices de comparaciones, ésta no da una información certera de la incertidumbre a nivel discreto de cada criterio o cada alternativa. 


\begin{tabular}{|c|c|c|c|c|c|c|}
\hline Criterios y Subcriterios & $1^{a}$ & $2^{\mathbf{a}}$ & $3^{a}$ & $4^{a}$ & $\mathbf{5}^{\mathbf{a}}$ & c.v. \\
\hline Económicos & $\begin{array}{l}0,17 \\
(0,109)\end{array}$ & $\begin{array}{l}0,17 \\
(0,105)\end{array}$ & $\begin{array}{l}0,24 \\
(0,068)\end{array}$ & $\begin{array}{l}0,28 \\
(0,036)\end{array}$ & $\begin{array}{l}0,17 \\
(0,107)\end{array}$ & 0,051 \\
\hline - Posibilidad de Sanción administrativa & $\begin{array}{l}0,66 \\
(0,104)\end{array}$ & $\begin{array}{l}0,64 \\
(0,094)\end{array}$ & $\begin{array}{l}0,57 \\
(0,104)\end{array}$ & $\begin{array}{l}0,62 \\
(0,083)\end{array}$ & $\begin{array}{l}0,68 \\
(0,019)\end{array}$ & 0,042 \\
\hline - Rendimiento & $\begin{array}{l}0,22 \\
(0,104)\end{array}$ & $\begin{array}{l}0,27 \\
(0,094)\end{array}$ & $\begin{array}{l}0,29 \\
(0,104)\end{array}$ & $\begin{array}{l}0,22 \\
(0,083)\end{array}$ & $\begin{array}{l}0,20 \\
(0,019)\end{array}$ & 0,038 \\
\hline - Mecanizable & $\begin{array}{l}0,12 \\
(0,104)\end{array}$ & $\begin{array}{l}0,09 \\
(0,094)\end{array}$ & $\begin{array}{l}0,14 \\
(0,104)\end{array}$ & $\begin{array}{l}0,16 \\
(0,083)\end{array}$ & $\begin{array}{l}0,12 \\
(0,019)\end{array}$ & 0,026 \\
\hline Ambientales & $\begin{array}{l}0,21 \\
(0,109)\end{array}$ & $\begin{array}{l}0,22 \\
(0,105)\end{array}$ & $\begin{array}{l}0,11 \\
(0,068)\end{array}$ & $\begin{array}{l}0,10 \\
(0,036)\end{array}$ & $\begin{array}{l}0,23 \\
(0,107)\end{array}$ & 0,063 \\
\hline - Erosión & $\begin{array}{l}0,80 \\
(-)\end{array}$ & $\begin{array}{l}0,75 \\
(-)\end{array}$ & $\begin{array}{l}0,75 \\
(-)\end{array}$ & $\begin{array}{l}0,75 \\
(-)\end{array}$ & $\begin{array}{l}0,75 \\
(-)\end{array}$ & 0,022 \\
\hline - Biodiversidad & $\begin{array}{l}0,20 \\
(-)\end{array}$ & $\begin{array}{l}0,25 \\
(-)\end{array}$ & $\begin{array}{l}0,25 \\
(-)\end{array}$ & $\begin{array}{l}0,25 \\
(-)\end{array}$ & $\begin{array}{l}0,25 \\
(-)\end{array}$ & 0,022 \\
\hline Sociales & $\begin{array}{l}0,06 \\
(0,109)\end{array}$ & $\begin{array}{l}0,06 \\
(0,105)\end{array}$ & $\begin{array}{l}0,06 \\
(0,068)\end{array}$ & $\begin{array}{l}0,06 \\
(0,036)\end{array}$ & $\begin{array}{l}0,06 \\
(0,107)\end{array}$ & 0 \\
\hline - Recreo & $\begin{array}{l}0,11 \\
(0,041)\end{array}$ & $\begin{array}{l}0,13 \\
(0,041)\end{array}$ & $\begin{array}{l}0,07 \\
(0,022)\end{array}$ & $\begin{array}{l}0,09 \\
(0,025)\end{array}$ & $\begin{array}{l}0,09 \\
(0,041)\end{array}$ & 0,023 \\
\hline - Otros usos & $\begin{array}{l}0,18 \\
(0,041)\end{array}$ & $\begin{array}{l}0,21 \\
(0,041)\end{array}$ & $\begin{array}{l}0,10 \\
(0,022)\end{array}$ & $\begin{array}{l}0,18 \\
(0,025)\end{array}$ & $\begin{array}{l}0,14 \\
(0,041)\end{array}$ & 0,043 \\
\hline - Cercanía a viviendas & $\begin{array}{l}0,71 \\
(0,041)\end{array}$ & $\begin{array}{l}0,66 \\
(0,041)\end{array}$ & $\begin{array}{l}0,72 \\
(0,022)\end{array}$ & $\begin{array}{l}0,75 \\
(0,025)\end{array}$ & $\begin{array}{l}0,77 \\
(0,041)\end{array}$ & 0,042 \\
\hline Técnicos & $\begin{array}{l}0,56 \\
(0,109)\end{array}$ & $\begin{array}{l}0,55 \\
(0,105)\end{array}$ & $\begin{array}{l}0,58 \\
(0,068)\end{array}$ & $\begin{array}{l}0,55 \\
(0,036)\end{array}$ & $\begin{array}{l}0,54 \\
(0,107)\end{array}$ & 0,015 \\
\hline - Altura de matorral & $\begin{array}{l}0,50 \\
(0,059)\end{array}$ & $\begin{array}{l}0,49 \\
(0,098)\end{array}$ & $\begin{array}{l}0,50 \\
(0,030)\end{array}$ & $\begin{array}{l}0,48 \\
(0,030)\end{array}$ & $\begin{array}{l}0,49 \\
(0,036)\end{array}$ & 0,008 \\
\hline - Carga de matorral & $\begin{array}{l}0,33 \\
(0,059)\end{array}$ & $\begin{array}{l}0,31 \\
(0,098)\end{array}$ & $\begin{array}{l}0,32 \\
(0,030)\end{array}$ & $\begin{array}{l}0,32 \\
(0,030)\end{array}$ & $\begin{array}{l}0,37 \\
(0,036)\end{array}$ & 0,023 \\
\hline - Necesidad de poda & $\begin{array}{l}0,08 \\
(0,059)\end{array}$ & $\begin{array}{l}0,07 \\
(0,098)\end{array}$ & $\begin{array}{l}0,11 \\
(0,030)\end{array}$ & $\begin{array}{l}0,08 \\
(0,030)\end{array}$ & $\begin{array}{l}0,09 \\
(0,036)\end{array}$ & 0,015 \\
\hline - Cercanía del turno & $\begin{array}{l}0,09 \\
(0,059)\end{array}$ & $\begin{array}{l}0,14 \\
(0,098)\end{array}$ & $\begin{array}{l}0,08 \\
(0,030)\end{array}$ & $\begin{array}{l}0,12 \\
(0,030)\end{array}$ & $\begin{array}{l}0,05 \\
(0,036)\end{array}$ & 0,035 \\
\hline
\end{tabular}

Tabla 2.- Peso e inconsistencias (entre paréntesis) de los diferentes criterios en distintas repeticiones (C.V.: Coeficiente de Variación)

\begin{tabular}{|c|c|c|c|c|c|c|}
\hline Criterios y Subcriterios & P1 & P 2 & P 3 & P 4 & P 5 & P 6 \\
\hline \multicolumn{7}{|l|}{ Económicos } \\
\hline - Posibilidad de Sanción administrativa & $\begin{array}{l}0,202 \\
(0,032)\end{array}$ & $\begin{array}{l}0,050 \\
(0,032)\end{array}$ & $\begin{array}{l}0,047 \\
(0,032)\end{array}$ & $\begin{array}{l}0,168 \\
(0,032)\end{array}$ & $\begin{array}{l}0,185 \\
(0,032)\end{array}$ & $\begin{array}{l}0,348 \\
(0,032)\end{array}$ \\
\hline - Rendimiento & $\begin{array}{l}0,009 \\
(0,037)\end{array}$ & $\begin{array}{l}0,379 \\
(0,037)\end{array}$ & $\begin{array}{l}0,211 \\
(0,037)\end{array}$ & $\begin{array}{l}0,155 \\
(0,037)\end{array}$ & $\begin{array}{l}0,205 \\
(0,037)\end{array}$ & $\begin{array}{l}0,041 \\
(0,037)\end{array}$ \\
\hline - Mecanizable & $\begin{array}{l}0,009 \\
(0,097)\end{array}$ & $\begin{array}{l}0,356 \\
(0,097)\end{array}$ & $\begin{array}{l}0,307 \\
(0,097)\end{array}$ & $\begin{array}{l}0,115 \\
(0,097)\end{array}$ & $\begin{array}{l}0,115 \\
(0,097)\end{array}$ & $\begin{array}{l}0,098 \\
(0,097)\end{array}$ \\
\hline \multicolumn{7}{|l|}{ Ambientales } \\
\hline - Erosión & $\begin{array}{l}0,04 \\
(0,094)\end{array}$ & $\begin{array}{l}0,295 \\
(0,094)\end{array}$ & $\begin{array}{l}0,144 \\
(0,094)\end{array}$ & $\begin{array}{l}0,144 \\
(0,094)\end{array}$ & $\begin{array}{l}0,200 \\
(0,094)\end{array}$ & $\begin{array}{l}0,176 \\
(0,094)\end{array}$ \\
\hline - Biodiversidad & $\begin{array}{l}0,024 \\
(0,004)\end{array}$ & $\begin{array}{l}0,306 \\
(0,004)\end{array}$ & $\begin{array}{l}0,285 \\
(0,004)\end{array}$ & $\begin{array}{l}0,285 \\
(0,004)\end{array}$ & $\begin{array}{l}0,06 \\
(0,004)\end{array}$ & $\begin{array}{l}0,039 \\
(0,004)\end{array}$ \\
\hline \multicolumn{7}{|l|}{ Sociales } \\
\hline - Recreo & $\begin{array}{l}0,072 \\
(0,022)\end{array}$ & $\begin{array}{l}0,516 \\
(0,022)\end{array}$ & $\begin{array}{l}0,124 \\
(0,022)\end{array}$ & $\begin{array}{l}0,096 \\
(0,022)\end{array}$ & $\begin{array}{l}0,096 \\
(0,022)\end{array}$ & $\begin{array}{l}0,096 \\
(0,022)\end{array}$ \\
\hline - Otros usos & $\begin{array}{l}0,155 \\
(0,021)\end{array}$ & $\begin{array}{l}0,01 \\
(0,021)\end{array}$ & $\begin{array}{l}0,197 \\
(0,021)\end{array}$ & $\begin{array}{l}0,244 \\
(0,021)\end{array}$ & $\begin{array}{l}0,197 \\
(0,021)\end{array}$ & $\begin{array}{l}0,197 \\
(0,021)\end{array}$ \\
\hline - Cercanía a viviendas & $\begin{array}{l}0,280 \\
(0,021)\end{array}$ & $\begin{array}{l}0,530 \\
(0,021)\end{array}$ & $\begin{array}{l}0,048 \\
(0,021)\end{array}$ & $\begin{array}{l}0,048 \\
(0,021)\end{array}$ & $\begin{array}{l}0,048 \\
(0,021)\end{array}$ & $\begin{array}{l}0,048 \\
(0,021)\end{array}$ \\
\hline \multicolumn{7}{|l|}{ Técnicos } \\
\hline - Altura de matorral & $\begin{array}{l}0,233 \\
(0,033)\end{array}$ & $\begin{array}{l}0,008 \\
(0,033)\end{array}$ & $\begin{array}{l}0,098 \\
(0,033)\end{array}$ & $\begin{array}{l}0,206 \\
(0,033)\end{array}$ & $\begin{array}{l}0,098 \\
(0,033)\end{array}$ & $\begin{array}{l}0,356 \\
(0,033)\end{array}$ \\
\hline - Carga de matorral & $\begin{array}{l}0,072 \\
(0,031)\end{array}$ & $\begin{array}{l}0,055 \\
(0,031)\end{array}$ & $\begin{array}{l}0,066 \\
(0,031)\end{array}$ & $\begin{array}{l}0,157 \\
(0,031)\end{array}$ & $\begin{array}{l}0,181 \\
(0,031)\end{array}$ & $\begin{array}{l}0,470 \\
(0,031)\end{array}$ \\
\hline - Necesidad de poda & $\begin{array}{l}0,107 \\
(0,023)\end{array}$ & $\begin{array}{l}0,107 \\
(0,023)\end{array}$ & $\begin{array}{l}0,107 \\
(0,023)\end{array}$ & $\begin{array}{l}0,382 \\
(0,023)\end{array}$ & $\begin{array}{l}0,107 \\
(0,023)\end{array}$ & $\begin{array}{l}0,190 \\
(0,023)\end{array}$ \\
\hline - Cercanía del turno & $\begin{array}{l}0,056 \\
(0,097)\end{array}$ & $\begin{array}{l}0,125 \\
(0,097)\end{array}$ & $\begin{array}{l}0,099 \\
(0,097)\end{array}$ & $\begin{array}{l}0,498 \\
(0,097)\end{array}$ & $\begin{array}{l}0,158 \\
(0,097)\end{array}$ & $\begin{array}{l}0,064 \\
(0,097)\end{array}$ \\
\hline
\end{tabular}

Tabla 3.- Pesos e inconsistencias (entre paréntesis) de cada una de las seis alternativas (parcelas) bajo cada criterio 


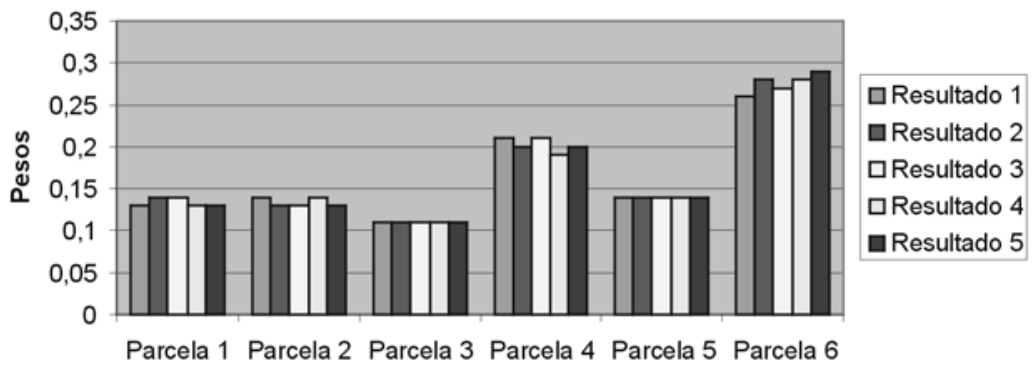

Figura 1.- Resultado de la Interacción de las repeticiones de los pesos de los criterios con los pesos de las alternativas

Parcela 1 Parcela 2 Parcela 3 Parcela 4 Parcela 5 Parcela 6 bajo cada criterio Alternativas

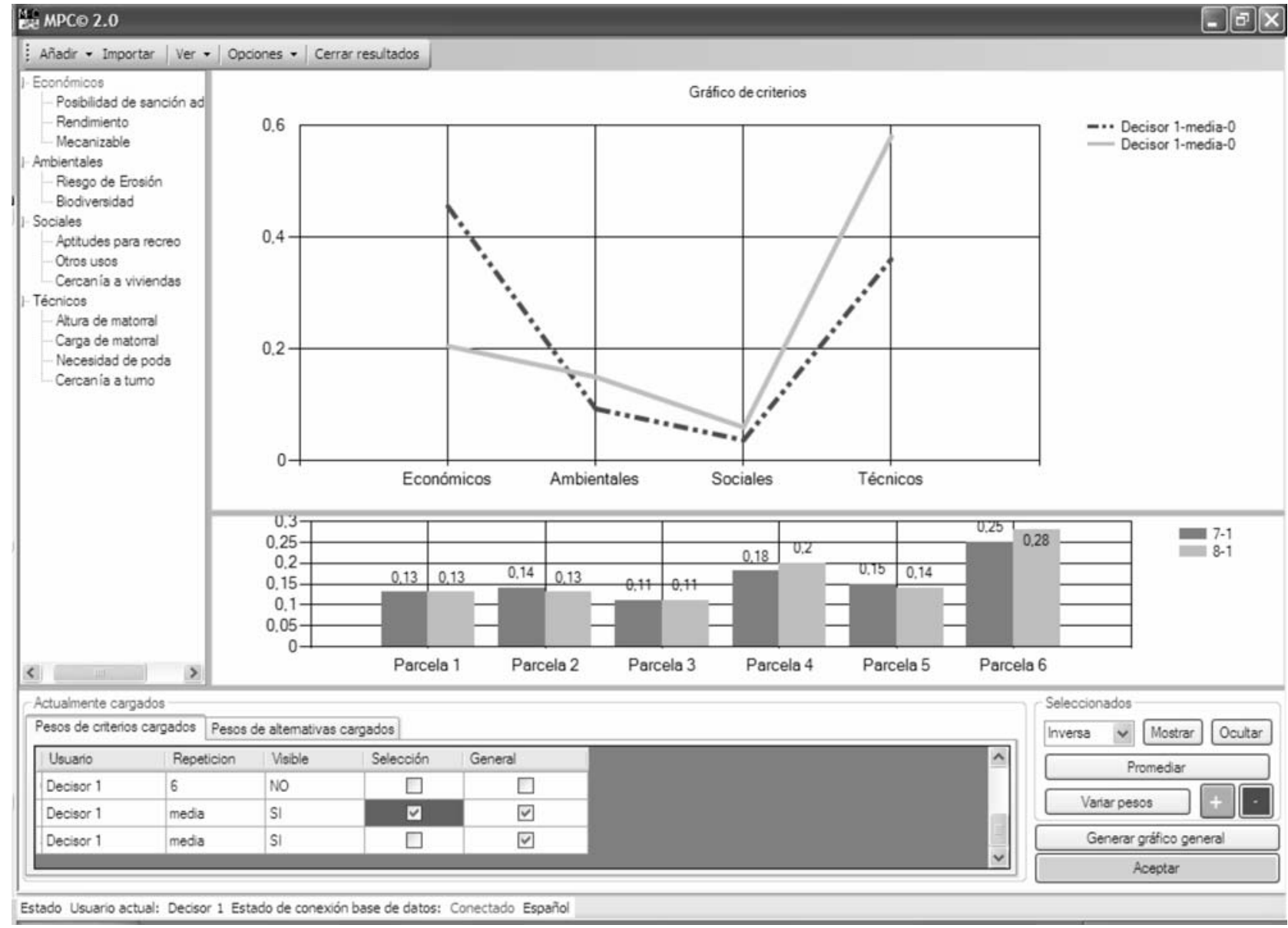

Figura 1.- Ejemplo de análisis de sensibilidad, donde se ha aumentado el peso del criterio económico un $25 \%$. En la gráfica de barras se observa que no cambia la jerarquía de parcelas en la serie de color gris oscuro, con respecto a la misma serie sin variar, de color gris mas claro

Según los resultados obtenidos (Figura 1), la parcela 6 es la que más peso ha obtenido, seguida de la parcela 4, por lo que éstas deberían ser las desbrozadas en el presente año. Más complicado resulta elegir la tercera parcela a ser desbrozada, pero como el resto deberán ser desbrozadas en años posteriores, siempre se podrían establecer en el futuro nuevas repeticiones con MPC $\odot$ antes de proceder a determinar su jerarquía. De esta manera, además se estaría retroalimentando la decisión, puesto que las características de las parcelas pueden ser cambiantes en el tiempo.
Con respecto al análisis de sensibilidad, el software utilizado permite variar los pesos obtenidos y observar la variación de los resultados finales en tiempo real. El método aplicado por MPC $\odot 2.0$ es el de variación proporcional, por lo que los resultados de este análisis han de ser cautelosamente estudiados debido a lo ya mencionado anteriormente. En la Figura 2 se puede observar que, por ejemplo, al aumentar un $25 \%$ el peso del conjunto de criterios económicos establecido, la jerarquía no varía. El análisis de sensibilidad ayuda al decisor a determinar la estabilidad de los resultados al variar la importancia de los criterios. 


\section{Conclusiones}

MPC $\odot 2.0$ es una herramienta informática que ayuda a la aplicación del método AHP de manera sencilla y rápida por tener automatizado todo el proceso de cálculo, y por ofrecer la posibilidad de interaccionar los pesos obtenidos de los criterios y de las alternativas entre diferentes usuarios y diferentes repeticiones de los mismos, pudiendo verse en cada momento los cambios que subyacen de estas interacciones en la decisión final. Además, el análisis de sensibilidad que incorpora MPC $\odot 2.0$ ayuda al decisor a observar la variabilidad, en tiempo real, en los resultados generales cuando se varía el peso de algún criterio.

Por otra parte, un responsable de una decisión ha de manejar todos los resultados obtenidos en las diferentes repeticiones realizadas, y ha de hacer un diseño de una toma de decisiones basándose en la variabilidad temporal y en la de diferentes decisores, sobre todo en toma de decisiones con alto impacto, recomendándose en este caso la obtención de intervalos de confianza para cada criterio para la aplicación de inferencia estadística y optimización. Solo así podrá llegarse a un resultado justificado, ya que el análisis de sensibilidad muestra cierto grado de subjetividad. Además, el responsable ha de ser crítico con el esquema de criterios propuesto, así como con las alternativas participantes, y debe asegurarse de que los resultados sean razonables y considerar si existe controversia, inconsistencia o variabilidad de los mismos.

La versión MPC $\odot 1.0$ puede descargarse de la Web del grupo de investigación "Unidade de Xestión Forestal Sostible" (UXFS) de la Escuela Politécnica Superior de Lugo, de la Universidad de Santiago de Compostela: http://www.usc.es/uxfs/

Agradecimientos Este trabajo se enmarca dentro del Subproyecto "Decide" (PSS-310000-2009-23), como parte del Proyecto "Restauración y gestión forestal" (PSE-310000-2009-4) del Ministerio de Ciencia e Innovación, financiado por la Unión Europea mediante fondos FEDER.

\section{Bibliografía}

Buongiorno, J., Keith, J. (2003). Decision methods for forest resource management. Academic Press. 439 pp

Kangas, A., Kangas, J., Kurttila, M. (2001). Decision Support for forest management. Ed. Springer. $221 \mathrm{pp}$.

Kangas, J.; Kangas, A. (2005) Multiple criteria decision support in forest management-the approach, methods applied, and experiences gained. Forest Ecology and Management 207. pp 133-143
Gadow, K.V., Bredenkamp, B. (1992). Forest management. Academica. Pretoria. $151 \mathrm{pp}$.

Leskinen, P., Kangas, J. (1998). Analysing uncertainties of interval judgment data in multiple-criteria evaluation of forest plans. Silva Fennica 32, 4: pp 363-372.

Pena de Ladaga, S., Berger, A. (2006). Toma de decisiones en el sector agropecuario. Editorial Facultad Agronomía. Buenos Aires. 308 pp.

Pérez, F., Rojo, A. (2010). Apply the AHP by new free software called MPC for take decisions in forest management. IUFRO World Congress, Seoul 2010.

Saaty, T.L. (1980). The Analytic Hierarchy Process. McGraw-Hill. New York. 287 pp.

Saaty, T.L. (1990). Decision making for Leaders. The Analitic Herarchy Process for decision in a complex World. University of Pittsburgh. RWS Publications, Pittsburgh, USA. $292 \mathrm{pp}$.

Saltello, A., Ratto, M., Tarantola, S., Campolongo, F. (2006). Sensitivity analysis practices: Strategies for model-based inference. Reliability. Engineering and System Safety 91: pp $1109-1125$

Schmoldt, D.L., Kangas, J., Mendoza, G.A., Pesonen, M. (2001). The Analytic Hierarchy Process in natural resource and environmental decision making. Kluwer Academic Publishers. Dortrecht. 328 pp.

Schoner, B., Wedley, W. (2007). Ambiguous Criteria Weights in AHP: Consequences and Solutions. Faculty of Business Administration, Simon Fraser University, Burnaby, British Columhia, Canada VSA IS6.

Toskano, G.B., Bruno, G. (2000). El proceso de Análisis Jerárquico (AHP) como Herramienta para la Toma de Decisiones en la Selección de Proveedores. Disponible en:

http://sisbib.unmsm.edu.pe/Bibvirtual/monografias/Basic/tos kano_hg/contenido.htm/Cap2.pdf [12 abril, 2010].

Triantaphyllou, E., Sanchez, A. (1997). A sensitivity analysis approach for some deterministic multi-criteria decision making methods. Decision Sciences. 28, 1: 151-194.

Zanazzi, J.L. (2003). Anomalías y supervivencia en el método de toma de decisiones de Saaty. En: Problemas del Conocimiento en Ingeniería y Geología, Vol. I, Godoy, L.A. (Editor): 148-170 pp. 